\title{
Deficiency of PDK1 in osteoclasts delays fracture healing and repair
}

\author{
DONGLIANG XIAO $^{1^{*}}$, QUAN ZHOU $^{2,3^{*}}$, YIGUANG BAI $^{1 *}$, BAICHUAN CAO $^{1}$, \\ QIONG ZHANG ${ }^{4}$, GAOFENG ZENG $^{4}$ and SHAOHUI ZONG ${ }^{1,5}$
}

\author{
${ }^{1}$ Department of Spine Osteopathia, The First Affiliated Hospital of Guangxi Medical University; \\ ${ }^{2}$ Collaborative Innovation Center of Guangxi Biological Medicine, Guangxi Medical University, Nanning, Guangxi 530021; \\ ${ }^{3}$ Department of Emergency, The Hongqi Hospital Affiliated to Mudanjiang Medical University, Mudanjiang, Heilongjiang 157001; \\ ${ }^{4}$ College of Public Hygiene; ${ }^{5}$ Research Centre for Regenerative Medicine and Guangxi Key Laboratory of \\ Regenerative Medicine, Guangxi Medical University, Nanning, Guangxi 530021, P.R. China
}

Received December 9, 2019; Accepted May 12, 2020

DOI: 10.3892/mmr.2020.11209

\begin{abstract}
Bone fractures are common traumatic injuries of the musculoskeletal system. However, delayed union and non-union fractures are a major clinical problem that present significant socioeconomic burden to patients and the public health sector. The bone-resorbing osteoclasts and bone-forming osteoblasts serve important roles in the fracture repair/healing process. Osteoclast deficiency or decreased osteoblast activity negatively impacts fracture healing. We previously demonstrated that the specific deletion of the serine/threonine kinase 3 -phosphoinositide-dependent protein kinase 1 (PDKI) in osteoclasts leads to abrogated osteoclast formation and bone resorption in response to receptor activator of nuclear factor- $x \mathrm{~B}$ in vitro and protected mice against ovariectomized-induced bone loss and lipopolysaccharide-induced osteolysis in vivo. Given the importance of osteoclasts in fracture repair, we hypothesized that the specific loss of PDK1 in osteoclasts will alter the fracture healing process. Mice of tibial fracture were constructed, and tibial specimens were sampled at 7-, 14-, 21- and 28-days post-fracture to observe the effect of $P D K 1$ gene regulated osteoclasts on fracture healing process by X-ray radiography, microcomputed tomography scanning,
\end{abstract}

Correspondence to: Professor Shaohui Zong, Department of Spine Osteopathia, The First Affiliated Hospital of Guangxi Medical University, 22 Shuangyong Road, Nanning, Guangxi 530021, P.R. China

E-mail: xiaohui3008@126.com

Professor Gaofeng Zeng, College of Public Hygiene, Guangxi Medical University, 22 Shuangyong Road, Nanning, Guangxi 530021, P.R. China

E-mail: 1685858372@qq.com

${ }^{*}$ Contributed equally

Key words: osteoclasts, gene knockout, bone fracture, callus, 3-phosphoinositide-dependent protein kinase 1 histomorphological staining and biomechanical testing. The present study revealed, using the tibial fracture model, that the specific deletion of the PDK1 gene in osteoclasts impeded the fracture healing process by delaying the resorption of the cartilaginous callus and subsequent remodeling of immature woven bone to structurally and mechanically ensure lamellar bone is stronger. No effect on osteoblast bone formation and osteogenesis was observed, thus indicating that delayed fracture healing is primarily due to defective osteoclast activity. These results provide important clinical implications for the use of anti-resorptive agents, such as bisphosphonates, for the treatment of osteolytic conditions. Such anti-resorptive therapies may detrimentally delay fracture healing and repair.

\section{Introduction}

Bone fractures are common musculoskeletal disorders that are the result of trauma or fragility, and present as a significant public health issue and socioeconomic burden (1). Despite advances in treatment options, up to $10 \%$ of all fractures exhibit insufficient bone repair leading to delayed or non-healing fractures. These fractures lead to significant morbidity and a significant decrease in the quality of life of patients who are affected $(2,3)$. Fracture healing is a complex process and causes of delayed bone repair are manifold, and the underlying cellular and molecular mechanisms are still unknown $(4,5)$. Understanding the biological and biomechanical aspects of the repair process is crucial for clinicians to develop novel treatment and management strategies for ensuring optimal repair of injured bone.

Fracture repair is an intricately specialized and complex postnatal regenerative process consisting of multiple events that occur simultaneously and in succession, with the end goal to restore the damaged/injured bone to pre-injury cellular composition, structure, and a biomechanically sound state $(4,6-8)$. The fracture healing process can be simplified into overlapping biological phases encompassing an early inflammatory response phase, the cartilaginous calls and hard callus formation middle phase, and the late bone remodeling phase. Each phase is governed by the activities of respective cell types, including immune cells (early phase), cartilage 
forming chondrocytes (early-middle phases), bone resorbing osteoclasts (middle-late phases), and the bone forming osteoblasts (middle-late phases) $(6,8-11)$. The early inflammatory phase occurs immediately post-fracture with hematoma formation at the fracture site creating a microenvironment that favors repair. Recruitment of repair cells, predominantly mesenchymal stem cells, occurs and are induced to differentiate into chondrocytes. During endochondral ossification, chondrocytes form the semi-rigid soft or cartilaginous callus, which provides mechanical support to the fracture ends. The cartilaginous callus also acts as the template for the bony hard callus. As the chondrocytes become hypertrophic, the cartilaginous callus becomes mineralized and is invaded by the vasculature. This enables the infiltration of osteoclasts, which functions to resorb the mineralized cartilage, allowing osteoblasts to remodel it into hard callus of immature woven bone. This ultimately leads to the bridging of the fracture by woven bone and osteoblasts play a major role in the initial formation of the woven bone matrix. The transition from cartilaginous callus to hard callus and woven bone formation represents the most active phase of osteogenesis. The final phase is the remodeling phase in which the action of the osteoblast and osteoclast remodels the immature hard callus of woven bone into the stronger mature lamellar bone, yielding bone that should structurally and mechanically match to the pre-fractured bone $(6,8-10)$.

The core of fracture healing is the remodeling process, which involves osteoclastic bone resorption and osteoblastic bone formation (6). Maintaining a dynamic balance between the activities of osteoclasts and osteoblasts is necessary for effective and adequate bone repair (12). Thus, perturbations in osteoclast-mediated bone/cartilage resorption and osteoblast-mediated bone formation during the process of bone renewal will negatively impact the rate and quality of fracture repair compromising bone microarchitecture and biomechanical properties $(13,14)$. Previous studies have found that blocking osteoclast formation and/or activity delays fracture repair by impairing cartilaginous callus resorption and hard callus remodeling $(15,16)$. We have previously demonstrated that the specific deletion of 3-phosphoinositide-dependent protein kinase 1 (PDK1), a serine/threonine kinase and pivotal regulator of PI3K-Akt signaling in osteoclasts, impairs receptor activator of nuclear factor- $x \mathrm{~B}$ (RANKL)-induced osteoclast formation and bone resorption (17). Given the importance of osteoclasts in fracture repair, we hypothesized that the specific loss of $P D K 1$ in osteoclasts will alter the fracture healing process. In the present study, it was revealed, using the tibial fracture model, that the specific deletion of $P D K 1$ in osteoclasts impeded the fracture healing process by delaying the resorption of the cartilaginous callus and subsequent remodeling of immature woven bone to structurally and mechanically stronger lamellar bone.

\section{Materials and methods}

Materials. Calcein, Alizarin Red S, the tartrate-resistant acid phosphatase (TRAP) staining kit and the Modified Safranin O-Fast Green FCF (SO/FG) Cartilage staining kit was purchased from Sigma-Aldrich (Merck KGaA). The TIANamp Genomic RNA Extraction kit was purchased from Tiangen Biotech Co., Ltd., while TRIzol reagent and the RevertAid
First Strand cDNA Synthesis kit was obtained from Thermo Fisher Scientific, Inc. ELISA for murine glaprotein/osteocalcin (BGP/OC), procollagen I N-terminal propeptide (PINP), TRACP-5b and C-terminal telopeptide of type I collagen (CTX-I) were purchased from Cusabio Biotechnology Co., Ltd.

Animals and experimental groups. All mice were raised and maintained in the Laboratory Animal Center of Guangxi Medical University (China). Mice were individually housed at a controlled temperature $\left(22-26^{\circ} \mathrm{C}\right)$ and humidity $(50-60 \%)$ in ventilated cages with a 12 -h light/dark cycle, with availability of standard chow and fresh-water ad libitum. Osteoclast-specific knockout (KO) of the PDK1 gene was generated as described in our previous study. Male transgenic mice expressing Cre recombinase, under the transcriptional control of the Cathepsin $\mathrm{K}$ promoter $\left(C t s K^{C r e}\right)$ were mated with female

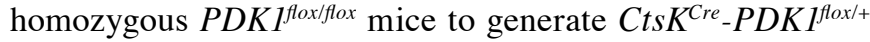
mice. Subsequently, $C t s K^{C r e}-P D K 1^{f l o x /+}$ mice were bred with $P D K 1^{\text {flox/+ }}$ mice to generate $C t s K^{C r e}-P D K 1^{\text {flox/flox }}$ mice (referred to as $P D K 1 \mathrm{cKO}$ ) and $C t s K^{C r e}-P D K 1^{+/+}$control wild-type (WT) mice. Genotyping was performed as previously described (17).

To generate the fracture model, 12-week-old WT and cKO mice ( $n=36$ per group; 72 mice in total) were anesthetized using sevoflurane. Penicillin sodium (200 U/g) was administered intraperitoneally to prevent infection following surgery. The left and right lower limb were shaved, and the skin disinfected with $0.5 \%$ iodophor. A longitudinal incision was made into the skin along the anterior-medial surface of the shaved lower limbs to expose the tibialis anterior muscle of the middle and upper segment of the tibia, which was then dissected. A total of 2, $0.5 \mathrm{~mm}$ Kirschner wires were inserted into the upper third of the tibia to guide the bone cut using small animal orthopedic scissors to create a transverse fracture. After fracture-end reduction, a Kirschner wire was surgically inserted from the tibial tubercle into the medullary canal for intramedullary fixation and stabilization of the fracture. The incision was sutured layer by layer. All mice were placed on heating beds and monitored until they had awoken from surgery. All mice were allowed to recover following surgery for 3-4 days with intraperitoneal administration of post-operative analgesics for pain relief for the first 2-3 days. A total of 24 mice (24 WT and 24 PDK1 cKO) were subsequently divided into 4 experimental groups; 7-, 14-, 21- and 28-days post-fracture. Prior to euthanasia, venous blood was extracted from the right ventricle in each mouse under anesthesia, from the 4 experimental groups, in order to analyze bone turnover markers using ELISA. Following blood collection, mice were sacrificed using cervical dislocation and the right tibias were dissected and processed for X-ray, micro computed tomography (CT) and histological analyses. The left tibias were removed, and the callus tissue was processed for total RNA extraction followed by reverse transcription-quantitative PCR (RT-qPCR) for analysis of gene expression.

To examine callus formation and the conversion of cartilage callus to hard callus, the remaining WT and cKO mice ( $n=12$ per group; 24 mice total) were subjected to Calcein Green-Alizarin Red S double labeling. Calcein Green $(25 \mathrm{mg} / \mathrm{kg})$ and Alizarin Red S (10 mg/kg) were administered intraperitoneally at 10- and 17-days post-surgery sequentially. Mice were sacrificed 21- and 28-days following post-fracture surgery, after which the right tibias were extracted for biome- 
chanical testing and the left tibias were extracted and processed for histological assessment of mineral apposition rate (MAR).

$X$-ray analysis. X-rays of the right tibia and fibula were obtained using a Faxitron MX20/DC2 (Faxitron Bioptics, LLC), equipped with an FPX-2 imaging system (Teledyne DALSA), with a voltage of $5.0 \mathrm{kV}$ and an exposure time of $6.0 \mathrm{sec}$. Blinded post-mortem radiographs were obtained for all samples in the 4 experimental groups and graded based on fracture line blurring. The degree of fracture line blurring was classified into three grades: clear, blurred and very blurred, as previously described $(18,19)$. All assessments were observed, discussed and collated by three independent researchers at the same time and recorded in detail, and the final results were used as the basis for subsequent downstream analysis.

MicroCT scanning. Tibial bone specimens were scanned using a high-resolution high-throughput compact microCT system (SCANCO $\mu$ CT 40; SCANCO Medical AG) and slices were analysed using the CT Evaluation Program (v5.0A, Scanco Medical). Scans performed at a voltage of $70 \mathrm{kV}$, current of $200 \mu \mathrm{A}$, and isotropic pixel size of $10 \mu \mathrm{m}$, with a $0.75-\mathrm{mm}$ thick aluminum filter for beam-hardening reduction. Morphometric analysis of callus bone volume percentage [callus bone volume/total volume (BV/TV), \%] and callus bone mineral density (callus BMD, $\mathrm{mg} / \mathrm{cm}^{3}$ ) were performed on reconstructed three-dimensional (3D) images in a region of interest, $1 \mathrm{~cm}$ centered around the fracture. The callus BMD reflects the porosity of the measured callus and the density of newly formed woven bone tissue.

Detection of bone turnover markers. Prior to mice being euthanized on days 7, 14, 21 and 28 post-surgery, venous blood was extracted from the right ventricle and serum was collected following centrifugation at $4^{\circ} \mathrm{C}, 1,500 \mathrm{x}$ g for $20 \mathrm{~min}$. Serum bone turnover markers, including BGP/OC (CSB-E06917m), PINP (CSB-E12775m), TRACP-5b (CSB-E08492m), and CTX-I (CSB-E12782m) were measured using the respective ELISA kits, according to the manufacturer's instructions. Serum samples were diluted in PBS (1:400) accordingly. So that optical density (OD) readings were within the range of the standard curve for the respective biochemical marker. OD readings were measured at a wavelength of $450 \mathrm{~nm}$ with a $650 \mathrm{~nm}$ reference filter, on a BioTek Elx800 Absorbance Microplate Spectrophotometer (BioTek Instruments, Inc.). The concentration of bone turnover in each group was calculated based on the generated standard curve.

Histology and histomorphometry. Following microCT scanning, tibial bone specimens were fixed in $4 \%$ paraformaldehyde for $48 \mathrm{~h}$ at $4^{\circ} \mathrm{C}$ and then decalcified in 10\% EDTA for 14 days at $4^{\circ} \mathrm{C}$. Decalcified bone tissues were embedded in paraffin, cut into 5- $\mu \mathrm{m}$ thick sections and then stained with hematoxylin and eosin $(\mathrm{H} \& \mathrm{E})$ at room temperature $\left(1826^{\circ} \mathrm{C}\right)$ for $30 \mathrm{~min}, \mathrm{TRAP}$ at $37^{\circ} \mathrm{C}$ for $1 \mathrm{~h}$, and Safranin $\mathrm{O}$ and Fast Green at room temperature $\left(1826^{\circ} \mathrm{C}\right)$ for $30 \mathrm{sec}$ and $20 \mathrm{~min}$, respectively. Histological images in five randomly selected fields were captured using an Olympus BX51 phase/fluorescence microscope (Olympus Corporation) at magnification, $\mathrm{x} 100$. TRAP-positive osteoclasts in the total callus were counted by two independent investigators. The total callus area $\left(\mathrm{mm}^{2}\right)$, cartilage area relative to the total callus, and hard callus area relative to total callus were calculated using SPOT Advanced Software (SPOT Imaging; Diagnostic Instruments, Inc.). Histomorphometry analysis of osteoclast parameters, including TRAP-positive osteoclast surface per bone surface (Oc.S/BS) and the number of TRAP-positve osteoclasts per trabecular bone perimeter (N.Oc/B.Pm) were carried out on TRAP-stained sections using the free Trap Histo Software (University of Liverpool) as previously described (20). Dynamic histomorphometry was performed on tibial bone samples from mice injected with Calcein Green-Alizarin Red double fluorescent probes. Following fixation in 10\% formaldehyde at $4^{\circ} \mathrm{C}$ for 3 days, undecalcified tibial bone tissues were dehydrated in an ascending ethanol series (70, 95, and twice with $100 \%$ ), washed with xylene and subsequently embedded in methyl methacrylate. The thin tissue sections $(10 \mu \mathrm{m})$ were cut by a Leica SM2500 microtome (Leica Microsystems $\mathrm{GmbH}$ ), and images were obtained using an Olympus BX51 phase/fluorescence microscope. The callus bone apposition rate (MAR, $\mu \mathrm{m} /$ day) was quantified in the following way: The distance between the fluorescence of Calcein Green used as the initial baseline(to stain bone formation 10 days after fracture) and the fluorescence of Alizarin Red used to reveal novel bone formation (to stain new bone formation 17 days after fracture) was measured and normalized by the time interval between the two injections. Three serial sections were examined and the mean value was analyzed, as previously described $(20,21)$.

Biomechanical analysis. Biomechanical testing was conducted on tibial bone specimens extracted from WT and cKO mice 21 and 28 days following post-fracture surgery. Following removal of the soft tissue-free full-length tibial shaft, the intramedullary nail was carefully removed, and the two ends of the tibial bone were fixed with matching fixtures, and then mounted on a torsion testing machine (Instron) for biomechanical torsion testing of the callus. The torsion angle rate was set to $0.2^{\circ} \mathrm{sec}$ and the rotational torsion testing was performed until failure. The maximum torque and torsional stiffness were determined using Bluehill Universal software (Instron). The maximum torque is the product of the force when the specimen is broken and the moment arm, and the torsional stiffness is a measure of the ability of the specimen to resist torsional deformation.

$R T-q P C R$. Following removal of the intramedullary nails, the RNA was extracted from the soft tissue-free tibial bone specimens from each experimental group using TRIzol reagent. The tibial bone specimens included normal bone tissues within $5 \mathrm{~mm}$ of the fracture ends and all callus tissues. Complementary DNA was reverse transcribed from 1-2 $\mu \mathrm{g}$ of extracted RNA using RevertAid First Strand cDNA Synthesis kit under the following conditions: $37^{\circ} \mathrm{C}$ for $5 \mathrm{~min}, 42^{\circ} \mathrm{C}$ for $60 \mathrm{~min}$ and $70^{\circ} \mathrm{C}$ for $10 \mathrm{~min}$. RT-qPCR was performed using the 7500 Real-Time PCR system (Applied Biosystems; Thermo Fisher Scientific, Inc.), with SYBR Green, cDNA template, and specific primers for osteoblast [osteocalcin (OCN), alkaline phosphatase (ALP), osteopontin (OPN) and type I collagen (COL1a2)] and osteoclast marker genes [Cathepsin K (CTSK), matrix metalloproteinase 9 (MMP-9), nuclear factor of activated t-cell cytoplasmic 1 (NFATc1) and TRAP (ACP5)], 
presented in Table SI. The following cycling conditions were used: initial denaturation at $95^{\circ} \mathrm{C}$ for $5 \mathrm{~min}$; then 40 repeated cycles at $95^{\circ} \mathrm{C}$ for $5 \mathrm{sec}, 60^{\circ} \mathrm{C}$ for $34 \mathrm{sec}, 72^{\circ} \mathrm{C}$ for $15 \mathrm{sec}$; and final extension at $72^{\circ} \mathrm{C}$ for $1 \mathrm{~min}$. mRNA levels were quantified using the $2^{-\Delta \Delta \mathrm{Cq}}$ method (22), and normalized to the internal reference gene $\beta$-actin.

Statistical analysis. All graphical data is presented as the mean \pm standard deviation (SD) of at least three independent biological repeats unless otherwise stated. The unpaired Student's t-test was used to compare differences between two groups and one-way ANOVA followed by the Tukey's post hoc test was used when comparing multiple groups, using SPSS software (v22.0; IBM Corp.). $\mathrm{P}<0.05$ was considered to indicate a statistically significant difference.

\section{Results}

Specific deletion of PDK1 in osteoclasts delays fracture healing. Previous studies have indicated that the process of fracture healing in normal mice is as follows: Hematoma formation 3 days following the fracture, with new bone formation starting at $\sim$ day 7 , this is followed by cartilaginous callus formation, which peaks at $\sim$ day 10 , followed by the transformation of the cartilaginous callus to hard callus $\sim$ days 10-14. At $\sim$ day 21, the surrounding callus begins to bridge the two ends of the fracture leading to fracture healing, and by day 28 , the fracture exhibits standard clinical healing $(23,24)$.

Using the aforementioned fracture healing characteristics as guidelines, the fracture healing process in $P D K 1 \mathrm{cKO}$ mice were examined using X-rays and microCT imaging. At 7 days following fracture surgery, the fracture line was clearly visible in both radiographs and microCT 3D images (Fig. 1A and B). Some calcified callus formation was observed in both WT and $P D K 1 \mathrm{cKO}$ mice, but no marked bone bridge between the two broken ends of fracture was identified. Furthermore, no significant difference in mineralized callus volume and BMD was observed between the WT and PDK1 cKO mice (Fig. 1C and D). At 14 days following fracture surgery, the fracture ends of both WT and PDK1 cKO tibial bones showed distinct hard callus formation with extension of the bone bridge that connects the fracture ends (Fig. 1A and B). However, compared with that in WT tibial bones, the fracture line was visible in $P D K 1$ cKO tibial bones, which we hypothesize is a consequence of a slower hematoma reabsorption. In addition, there was no significant difference in mineralized callus volume, but a lower callus BMD was identified in WT mice (Fig. 1C and D). At 21 days post-fracture surgery, the calcified callus at the fracture ends of WT tibial bones had largely been resorbed, with no visible fracture line (Fig. 1A and B). The hard callus bone surrounding the fracture exhibited signs of remodeling into plate-like cortical bone (Fig. 1B). On the other hand, large amounts of calcified callus were observed in the fracture ends of $P D K 1 \mathrm{cKO}$ tibial bones with apparent bridging callus between fracture ends similar to that observed in WT tibial bones on day 14 (Fig. 1A and B). Mineralized callus volume was significantly higher in WT tibial bones as compared with that in PDK1 cKO bones at day 21 (Fig. 1C). As with day 14, callus BMD at day 21 in WT tibial bones were lower compared with that in $P D K 1$ cKO tibial bones (Fig. 1D). Lastly, at 28 days following post-fracture surgery, the callus at the fracture site of WT tibial bone had completely healed with complete cortical bone remodeling (Fig. 1A and B). On the other hand, PDK1 cKO tibial bones exhibited callus characteristics similar to those in WT tibial bones observed on day 21 with calcified callus resorption, and cortical bridge remodeling (Fig. 1B). Mineralized callus volume was also significantly higher in WT mice compared with that in PDK1 cKO mice (Fig. 1C), whereas callus BMD was significantly lower in WT mice (Fig. 1D). The results observed at days 21 and 28 indicates that delayed healing, slower callus resorption and cortical bridge remodeling of the tibial bone fracture is observed in $P D K 1 \mathrm{cKO}$ mice. The detailed score of the blurry degree of fracture line is shown in Table SII. The delayed callus resorption and cortical bridge remodeling is likely to be the consequence of reduced osteoclast formation and activity, as a result of loss of PDK1.

Loss of PDK1 in osteoclasts compromises the biomechanical quality of the fracture callus. The biomechanical properties of the fracture callus was assessed at days 21 and 28 following fracture surgery and the results revealed that the fracture callus from $P D K 1 \mathrm{cKO}$ tibial bones were markedly weaker compared with that in WT mice at both days 21 and 28 following fracture surgery. The maximum torque and torsional stiffness were significantly decreased in $P D K 1 \mathrm{cKO}$ tibial bones when compared with that in WT tibial bones (Fig. 2A and B). These biomechanical results indicate that the specific loss of $P D K 1$ in osteoclasts negatively impacts the bone-callus microarchitecture during the fracture healing process, leading to compromised biomechanical quality.

Loss of PDK1 in osteoclasts delays callus resorption and remodeling. $\mathrm{H} \& \mathrm{E}$ and $\mathrm{SO} / \mathrm{FG}$ staining was performed on tibial bone sections to visualize general bone tissue and cartilage microarchitecture, respectively, during the fracture healing process. As shown in Fig. 3A and B, the progression of fracture healing was delayed in $P D K 1 \mathrm{cKO}$ mice. At 7 days post-fracture surgery, large amounts of undifferentiated mesenchyme and some proliferating chondrocytes accumulated at the broken end of the fracture in both WT and PDK1 cKO tibial bones. Most of the callus at this timepoint, in both experimental groups were organized hematoma, with some degree of cartilaginous callus. By day 14 post-fracture, large numbers of hypertrophic chondrocytes were present at the broken ends of the fracture, and transition from cartilaginous calluses to hard calluses were observed in both WT and PDK1 cKO groups (Fig. 3A and B). At 7- and 14- days post-fracture, no significant differences in the area of cartilaginous callus, hard callus and total callus were observed between the WT and PDK1 groups (Fig. 3C-E). At 21-days post-fracture, few chondrocytes were observed in the broken ends of the fracture in WT tibial bones. The hard callus in the WT tibial bone fracture site were gradually reabsorbed transitioning from immature woven to more mature lamellar bone structure. A large number of osteoblasts and osteocytes in the WT tibial bones were observed (Fig. 3A and B). By day 28, most of the hard callus in the WT tibial bones had been resorbed with the observation of mature lamellar cortical bone and medullary cavity. On the other hand, hypertrophic chondrocytes were the most abundant cells observed in the remaining cartilaginous callus and the hard callus at the fracture site 


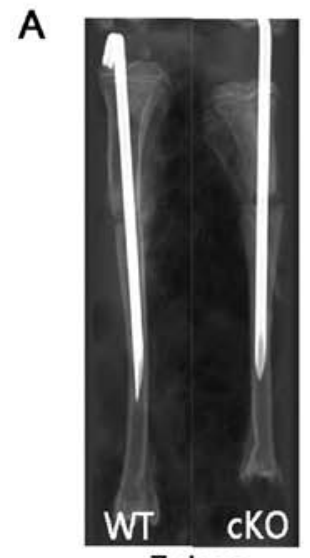

B

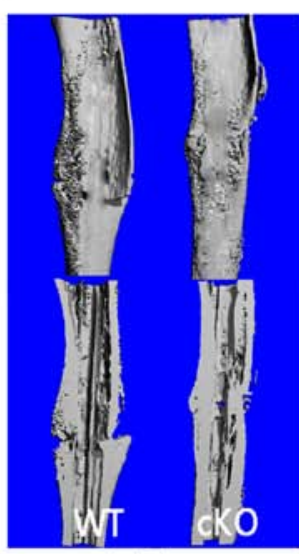

7 days

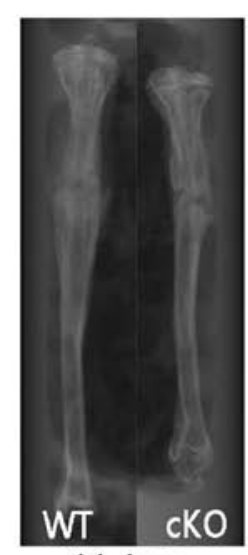

14 days

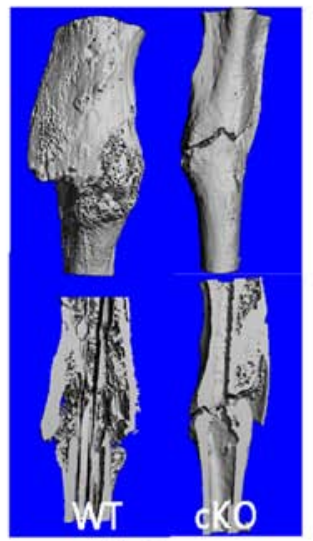

14 days

C

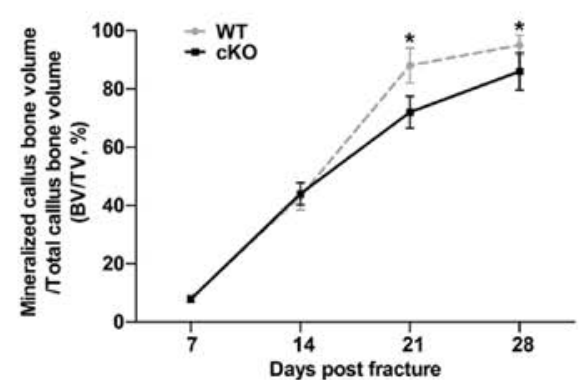

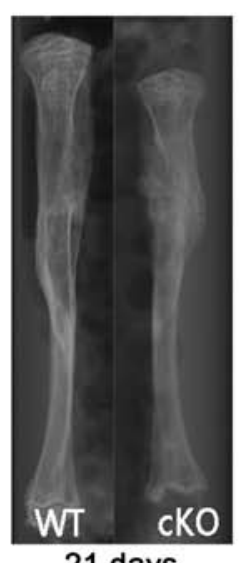

21 days

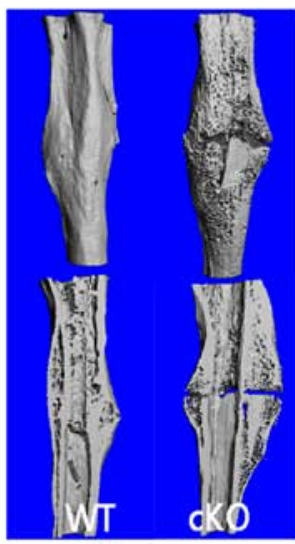

21 days

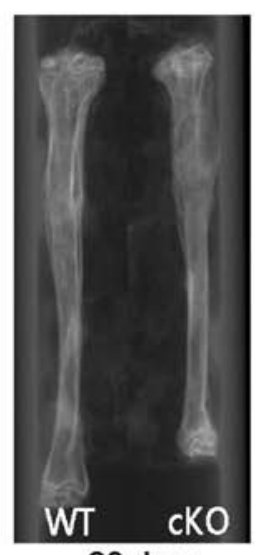

28 days

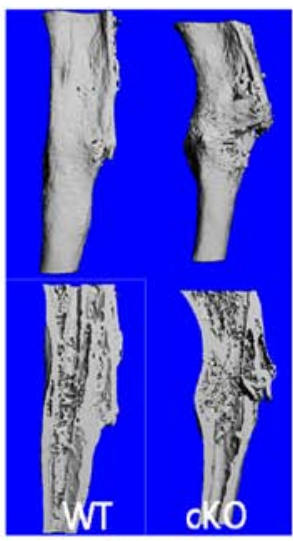

28 days

D

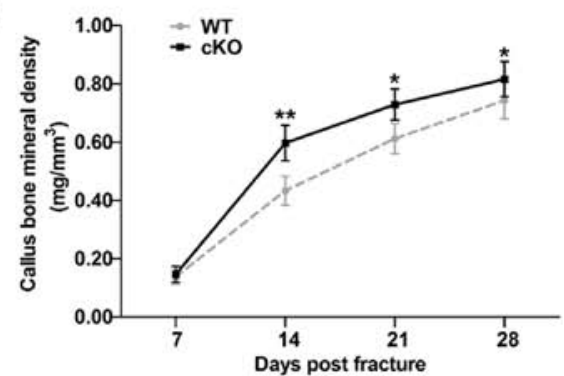

Figure 1. Deletion of the PDK1 gene in osteoclasts delays fracture union and repair. (A) Representative X-ray and (B) three-dimensional microCT images of the fractured tibia in WT and PDK1 cKO mice at 7, 14, 21 and 28 days post-fracture. (C and D) Morphometric analysis of the changes in mineralized callus $\mathrm{BV} / \mathrm{TV}$, and callus bone mineral density were measured. Data are presented as mean $\pm \mathrm{SD} .{ }^{*} \mathrm{P}<0.05,{ }^{* *} \mathrm{P}<0.01 . \mathrm{BV}$, bone volume; TV, tissue volume; WT, wild-type; cKO, conditional knockout; PDK1 cKO, Ctsk ${ }^{C r e}-P D K 1^{f o x / f o x}$; PDK1, 3-phosphoinositide-dependent protein kinase 1.
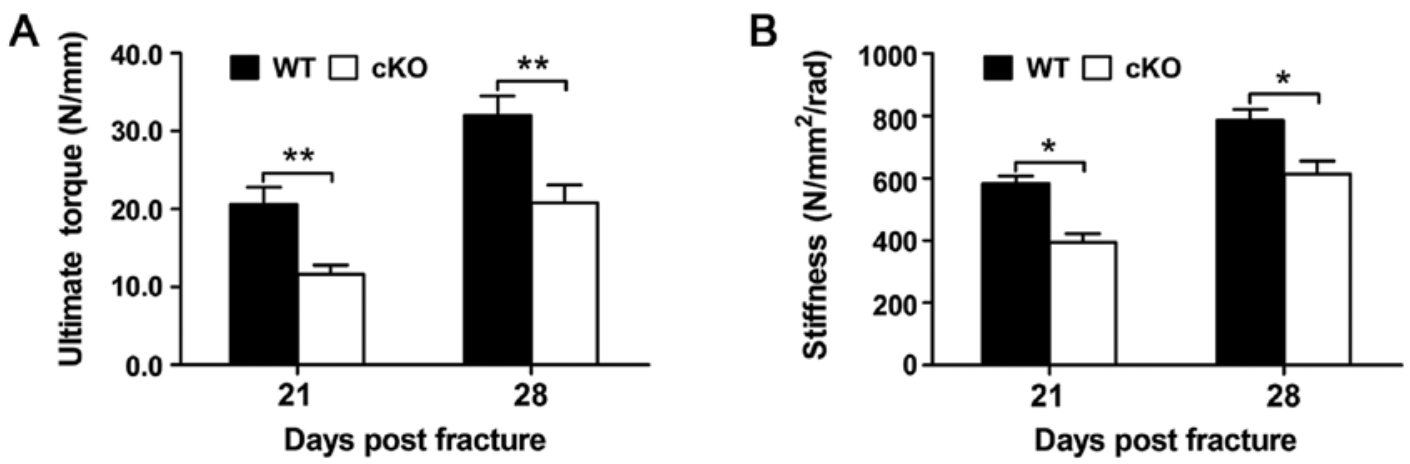

Figure 2. Loss of PDK1 in osteoclasts compromises bone biomechanical properties. Biomechanical analysis of (A) maximum/ultimate torque and (B) stiffness of the tibial fracture callus at 21 - and 28 -days post-fracture. Data are presented as mean $\pm \mathrm{SD}$. ${ }^{*} \mathrm{P}<0.05,{ }^{* *} \mathrm{P}<0.01$. WT, wild-type; cKO, conditional knockout; PDK1 cKO, Ctsk ${ }^{C r e}$-PDK1 $1^{\text {floxfflox}}$; PDK1, 3-phosphoinositide-dependent protein kinase 1. 
A
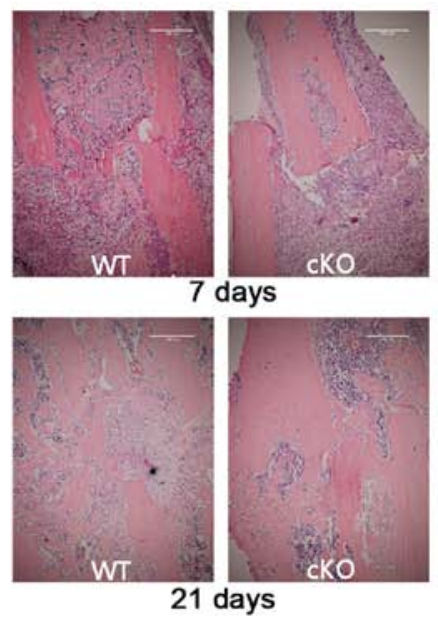

B

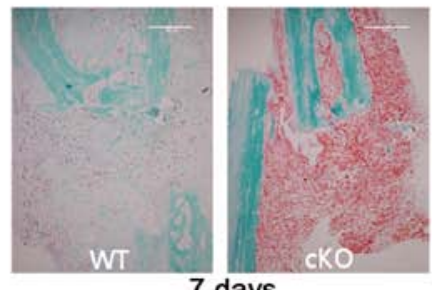

7 days

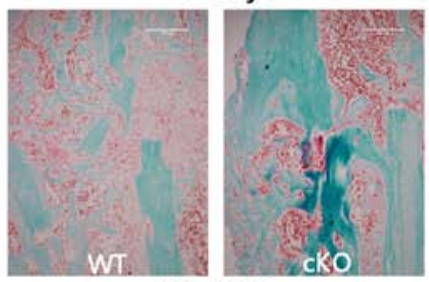

21 days
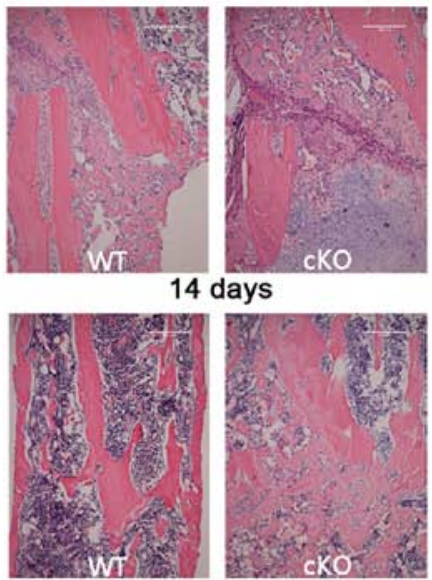

28 days

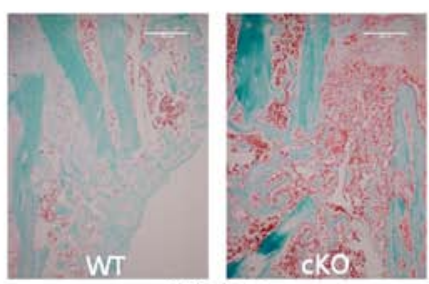

14 days

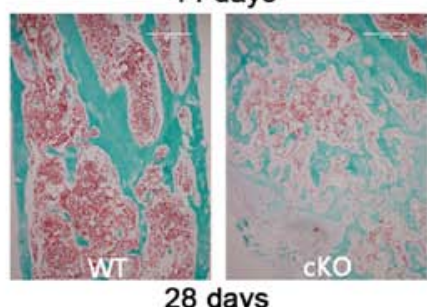

28 days
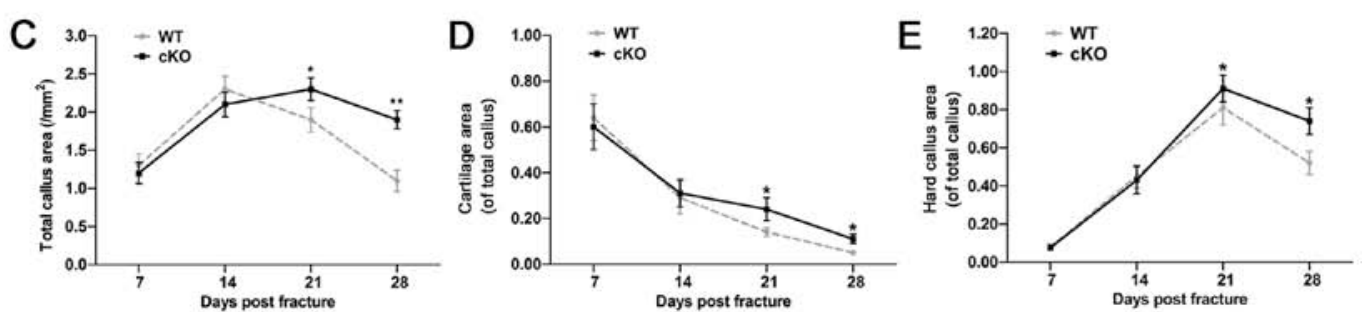

Figure 3. PDK1 deletion in osteoclasts delays cartilaginous callus resorption and remodeling. (A) Representative histological hematoxylin and eosin, and (B) Safranin O-Fast Green FCF stained sections of the tibial fracture callus at 7, 14, 21- and 28-days post-fracture. (C-E) Quantitative histomorphometric measurement of total callus area, cartilaginous callus area (with respect to total callus area) and hard callus area (with respect to total callus area) of WT and PDK1cKO tibial bones at each time point. Data are presented as mean $\pm \mathrm{SD} .{ }^{*} \mathrm{P}<0.05,{ }^{* *} \mathrm{P}<0.01$. WT, wild-type; cKO, conditional knockout; PDK1 cKO, $C t s k^{C r e}-P D K 1^{f f o x f f o x}$; PDK1, 3-phosphoinositide-dependent protein kinase 1.

were mostly immature woven trabecular bone in the PDKl cKO group at 21 days post-fracture. Transition from immature woven bone to lamellar bone started at day 28 in the PDKI cKO group, with evidence of residual cartilaginous callus and large amounts of irregular trabecular bone within the medullary space. No cortical bone structure was observed (Fig. 3A and B). The cartilaginous callus, hard callus and total callus area in the cKO group were markedly greater compared with that in the WT group at both 21- and 28-days following fracture surgery (Fig. 3C-E). Together, these results suggest that the loss of $P D K 1$ in osteoclasts significantly delays callus resorption and bone remodeling during the fracture healing process.

Osteoclast deletion of PDK1 reduces osteoclast activity but not osteoblast bone formation activity. TRAP staining was also performed to examine osteoclast parameters in the fracture callus during the fracture healing process. From day 7 to 21 post-fracture, abundant numbers of TRAP ${ }^{+v e}$ multinucleated osteoclasts were observed in the fracture ends of WT tibial bones, which were predominantly localized to the hypertrophic zone of the cartilaginous callus and the trabecular surface of the hard callus (Fig. 4A). Morphometric quantitation of the N.Oc/B.Pm and Oc.S/BS (\%) further showed that peak osteoclast formation and activity occurred between days 14 and 21 (Fig. 4B and C). By day 28 , the number and activity of TRAP ${ }^{+v e}$ osteoclasts were significantly reduced, coinciding with the end of the fracture healing process. On the other hand, the number and activity of TRAP $^{\text {+ve }}$ osteoclasts in the fracture callus of PDK1 cKO tibial bones were significantly lower at all timepoints when compared with that in WT tibial bones (Fig. 4A-C). 
A
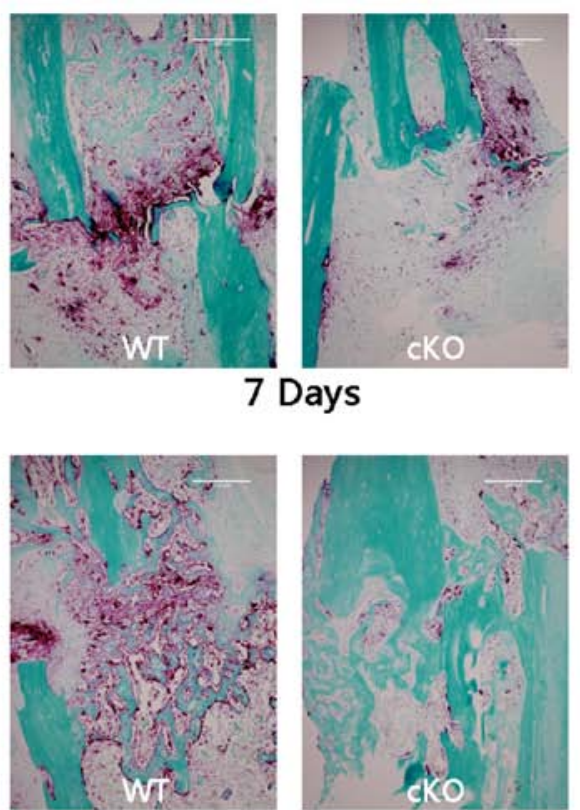

21 Days

B

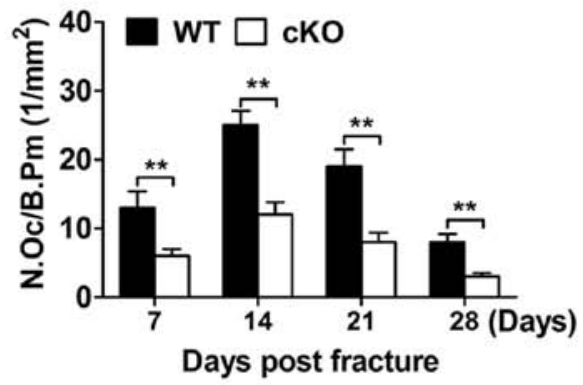

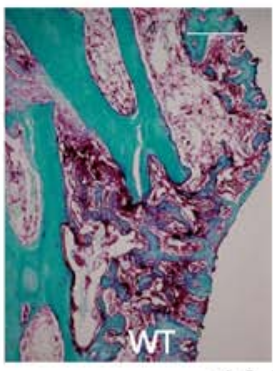

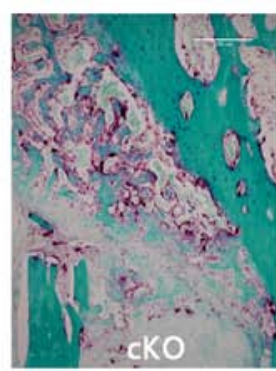

14 Days

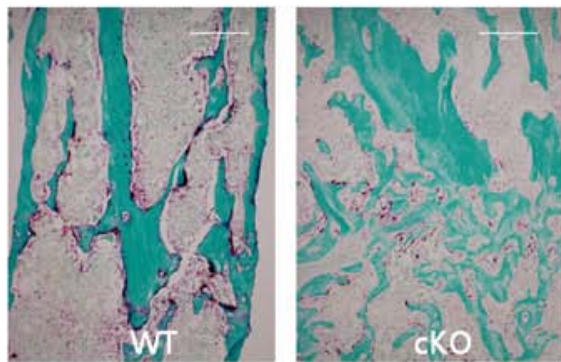

28 Days

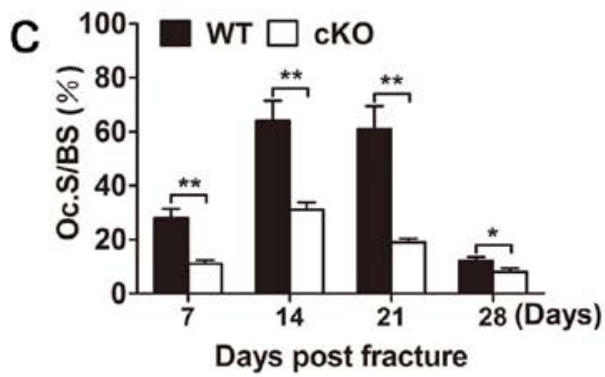

D

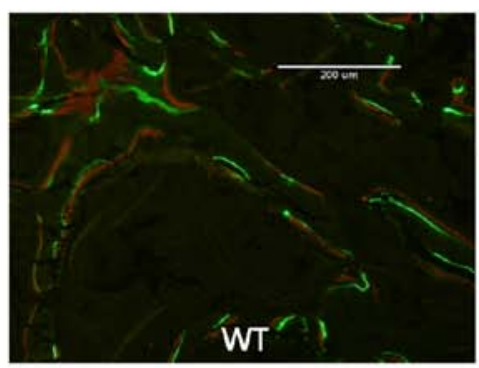

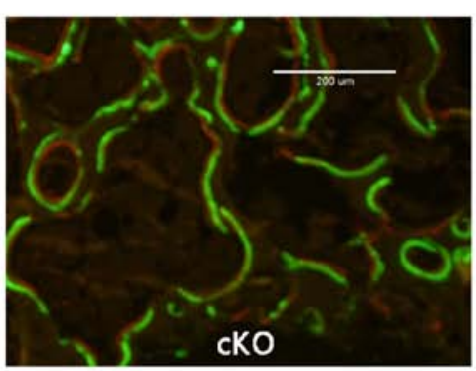

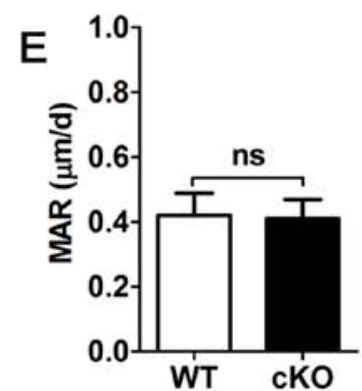

Figure 4. PDK1 is required for osteoclast formation and bone resorption, but not osteoblast bone formation. (A) Representative TRAP stained sections of tibial fracture callus in WT and PDK1 cKO mice 7, 14, 21- and 28-days post-fracture. (B and C) Quantitative histomorphometric analysis of the N.Oc/B.Pm and Oc.S/BS in the fracture callus were performed. (D and E) Representative Calcein-Alizarin Red S double stained sections of tibial fracture callus and associated dynamic histomorphometric measurement of bone mineral apposition rate in WT and PDK1 cKO mice. Fluorescence staining of irregular trabecular bone in new callus. Green fluorescence represents calcein and red fluorescence represents Alizarin Red S. Data are presented as mean $\pm \mathrm{SD}$. ${ }^{*} \mathrm{P}<0.05$, ${ }^{* *} \mathrm{P}<0.01$. ns, no significant difference; cKO, conditional knockout; PDK1 cKO, Ctsk ${ }^{C r e}$-PDK $1^{f f o x / f o x}$; WT, wild-type; PDK1, 3-phosphoinositide-dependent protein kinase 1; TRAP, tartrate-resistant acid phosphatase; Oc.S/BS, osteoclast surface per bone surface; N.Oc/B.Pm, number of osteoclasts per bone perimeter.

The MAR results showed no significant difference between WT and PDK1 cKO mice (Fig. 4D and E), indicating that osteoblast-mediated bone formation was not affected during the fracture healing process. Analysis of serum levels of bone turnover markers measured at days 7 , 14, 21 and 28 showed a significant reduction in bone resorption markers, such as TRAP and CTX-I in PDKl cKO mice (Fig. 5A and B). No change in bone formation markers of BGP and PINP were observed between WT and PDK1 cKO mice (Fig. 5C and D). Consistent with these serum results, RT-qPCR analysis of gene expression in callus tissues further showed a reduction in osteoclast marker genes, including CTSK, MMP-9, TRAP and NFATc1 (Fig. 6A-D). As with previous results, no change in the mRNA expression levels of osteoblast marker genes, such as OCN, ALP, OPN and COL1a2 was observed (Fig. 6E-H). It appears that osteoblast bone formation and osteogenesis was not affected. Collectively, these results provide further evidence that reduced osteoclast formation and activity, and not osteoblast activity, was responsible for the delayed cartilaginous callus resorption and bone remodeling during the fracture healing process. 

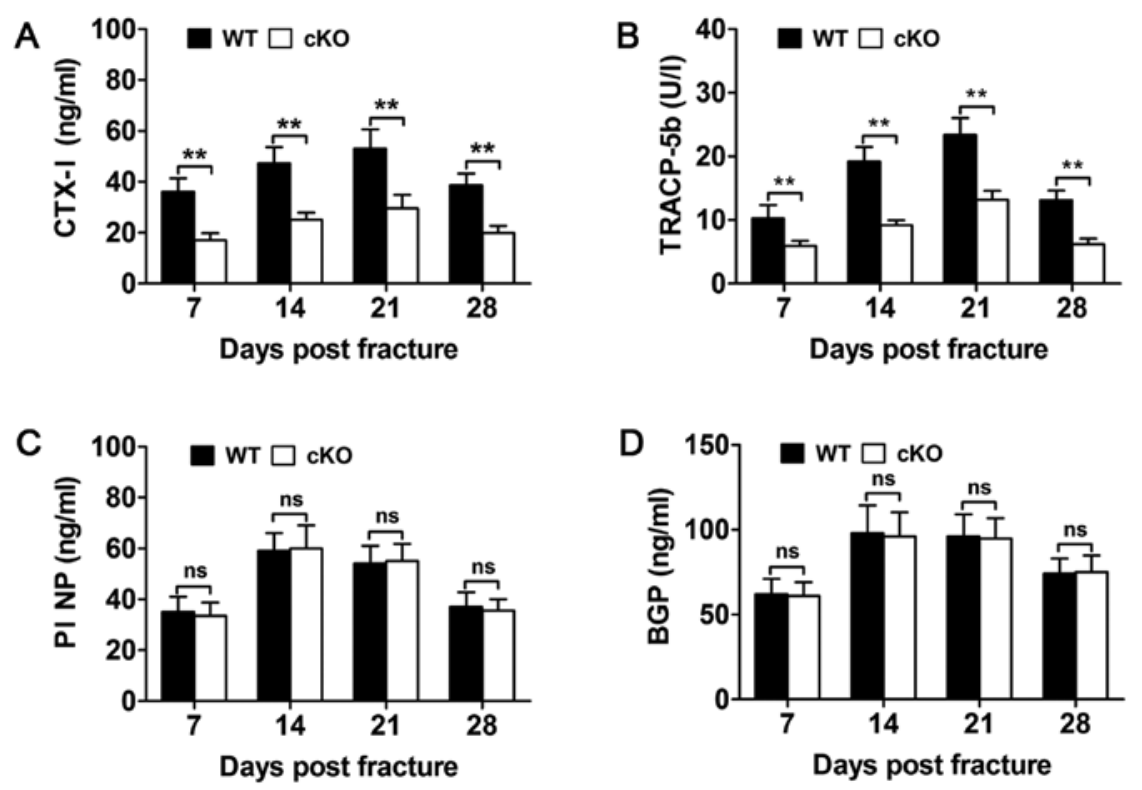

Figure 5. Osteoclast-specific deletion of $P D K 1$ impairs osteoclasts bone resorption, but not osteoblast bone formation. Changes in serum biochemical indices of bone resorption, including (B) TRACP5b and (A) CTX-I, and bone formation, such as (D) BGP/osteocalcin and (C) PINP at 7, 14, 21 and 28 days post-fracture in WT and $P D K 1 \mathrm{cKO}$ mice were measured using ELISA. Data presented as mean $\pm \mathrm{SD}$. ${ }^{* *} \mathrm{P}<0.01$. ns, no significant difference; cKO, conditional knockout; PDK1 cKO, Ctsk ${ }^{C r}$-PDKI $1^{f l o x f f l o x}$; WT, wild-type; PDK1, 3-phosphoinositide-dependent protein kinase 1; TRAP, tartrate-resistant acid phosphatase; CTx-I, C-terminal telopeptide of type I collagen; BGP, glaprotein; PINP, procollagen I N-terminal propeptide.
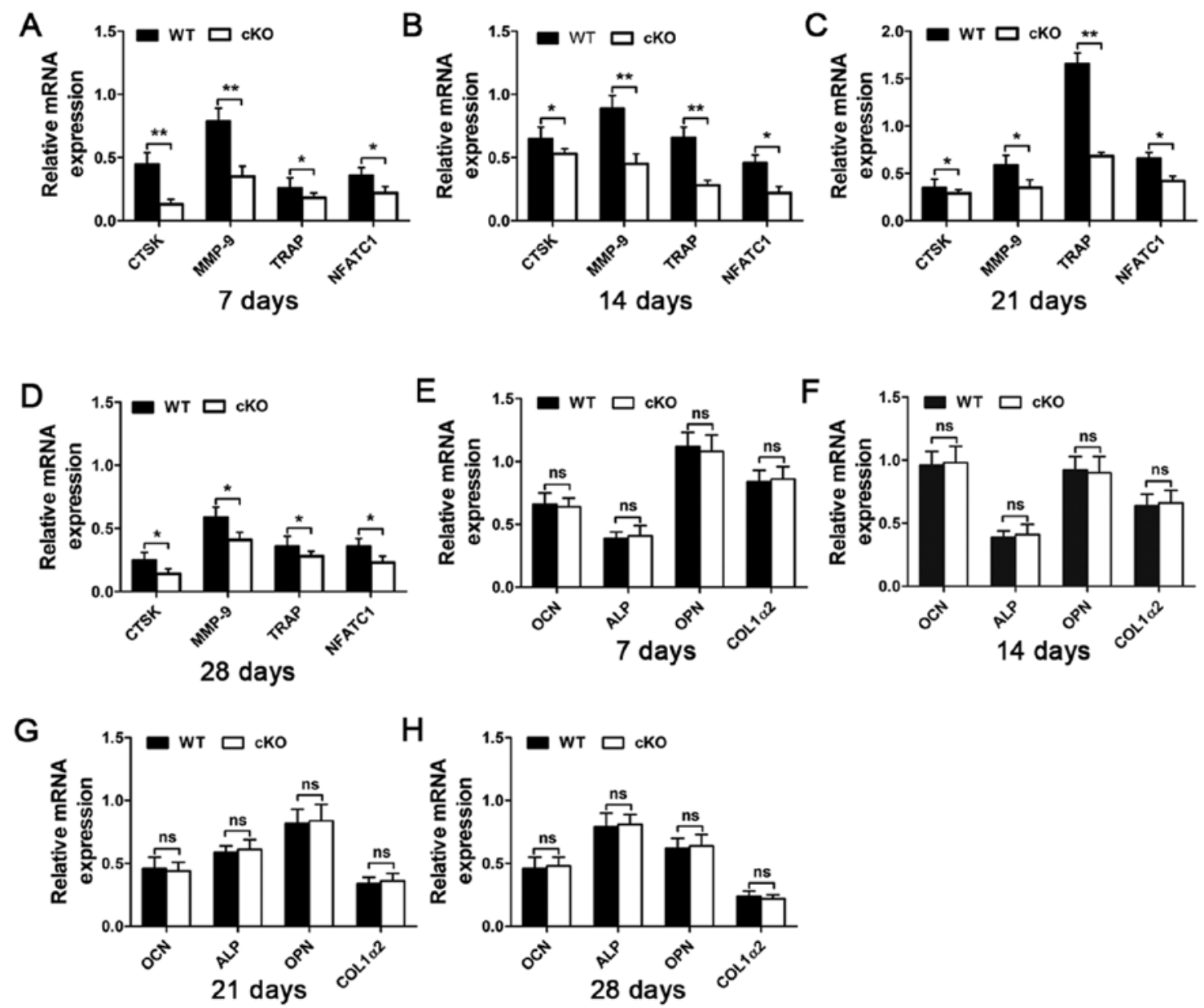

Figure 6. Osteoclasts marker gene expression level is downregulated following the deletion of PDK1 gene. The expression of (A-D) osteoclast marker genes, including CTSK, MMP-9, TRAP and NFATc1, and (E-H) osteoblast marker genes, including OCN, ALP, OPN and COL1 $\alpha 2$ at 7, 14, 21- and 28-days post-fracture were examined using reverse transcription-quantitative PCR. ${ }^{*} \mathrm{P}<0.05,{ }^{* *} \mathrm{P}<0.01$. ns, no significant difference; cKO, conditional knockout; $P D K 1 \mathrm{cKO}$, Ctsk ${ }^{\text {Cre }}-P D K 1^{f o x} / f \circ x_{\text {; }}$; WT, wild-type; PDK1, 3-phosphoinositide-dependent protein kinase 1; TRAP, tartrate-resistant acid phosphatase; CTSK, Cathepsin K; MMP-9, matrix metalloproteinase 9; NFATc1, nuclear factor of activated t-cell cytoplasmic 1; OCN, osteocalcin; ALP, alkaline phosphatase; OPN, osteopontin; COL1 22 , type I collagen. 


\section{Discussion}

Bone fracture healing is a complex, dynamic and multi-staged process necessary for the restoration of damaged bone to a functionally and biomechanically normal state. Both the bone resorbing osteoclasts and the bone forming osteoblasts play indispensable roles in the fracture healing process $(6,9,10,25,26)$. In our previous study, it was revealed, using cellular and biochemical methodologies, that the specific deletion of $P D K 1$ in osteoclasts impairs RANKL-induced osteoclast formation and bone resorption via the suppression of the Akt-GSK3 $\beta$-NFATc1 signaling pathway (17). Thus, we hypothesized that the specific loss of $P D K 1$ in osteoclasts will affect fracture healing. In the present study, using the $P D K 1$ $\mathrm{cKO}$ mice and the tibial fracture model, it was revealed that the specific deletion of $P D K 1$ in osteoclasts impeded the fracture healing process by delaying the resorption of the cartilaginous callus during the middle phase (14-21 days post-fracture), thereby hindering the remodeling of immature woven bone to mature lamellar bone during the late phase (21-28 days post-fracture)

As the present data has shown, osteoclasts play an important role in fracture healing process. Osteoclasts are predominantly involved in the middle and late phases of the fracture healing process and are typically responsible for the resorption and remodeling of cartilaginous soft callus for replacement with hard callus $(16,27)$. On the other hand, immune cells and chondrocytes play a more dominant role during the early phase of fracture repair $(4,6,28)$. As such, and despite significant decreases in osteoclasts in the fracture site of $P D K 1 \mathrm{cKO}$ tibial bones, significant differences were not observed in fracture repair between WT and $P D K 1$ cKO mice during the early phase (7-14 days post-fracture) of inflammation, hematoma development and cartilaginous callus formation. Callus volume and cartilaginous callus/hard callus relative to total callus, were similar between WT and PDK1 cKO mice during the early phase of fracture repair.

The middle and late phases (14-28 days post-fracture) of cartilaginous callus resorption, and remodeling of woven bone to lamellar bone are important processes during fracture repair. Previous genetic and pharmacological drug studies have shown that inhibition of osteoclast formation and/or bone resorption activity delays fracture healing by disrupting cartilage dissolution and remodeling, and increases fracture callus size $(15,16,27,29,30)$. On the other hand, increased osteoclast formation accelerated fracture healing (31). In the present study, X-rays and microCT 3D images from $P D K 1 \mathrm{cKO}$ mice revealed distinct fracture lines and increased callus size from 14-28 days post-fracture. Histological assessments of tibial bone sections from $P D K 1$ cKO mice further showed markedly decreased total numbers of TRAP ${ }^{+v e}$ osteoclasts near the chondro-osseous junctions at the fracture site, as well as the number of active osteoclasts on the bone surface, which was associated with delayed cartilaginous callus resorption and woven bone remodeling. Reduced osteoclast formation and activity was further confirmed by gene expression analyses of osteoclast marker genes, including NFATc1, TRAP, CTSK and MMP-9. Both CTSK and MMP-9 are important proteases required for the resorption process. In particular, it has been reported that MMP-9 is crucially involved in soft callus remodeling and hypertrophic chondrocyte apoptosis, with the lack of MMP-9 leading to delayed fracture healing $(32,33)$.

The amount of cartilaginous callus in fracture site of $P D K 1$ cKO tibial bones were comparable to WT mice in the early phase of fracture repair; however, there was considerably more cartilaginous callus that was not reabsorbed (greater overall callus bone volume and area with respect to total callus area) in the middle phase in $P D K 1 \mathrm{cKO}$ tibial bones, suggesting that the impediment of fracture repair was not due to the initial size of the formed cartilaginous callus but rather the resorption and remodeling of the cartilaginous callus to hard callus. This delay became more apparent during the late phase (28 days post-fracture) of fracture healing, with the total callus volume in the $P D K 1$ cKO group being significantly larger compared with that in the WT group. The larger callus area, bone volume and BMD observed at the middle and late phases of fracture repair was not due to increased osteoblast activity, as osteoblast MAR, serum bone formation markers (PINP and BGP), and osteoblast marker genes (OCN, ALP, OPN, COL1a2) were not affected by the loss of $P D K 1$ in osteoclasts. By this time most of the cartilaginous callus had been remodeled to hard callus in the $P D K 1$ cKO group; however, morphologically abundant immature woven trabecular bone was observed in the medullary of the tibial bones with no apparent cortical shell formation. In comparison, in the WT group, most of the immature woven trabecular bone had been remodeled to lamellar bone with cortical shell formation largely complete, displaying overall normal bone architecture. These results were similar to previous studies which examined the effects of bisphosphonates on fracture healing and repair $(14,34)$. Biomechanical analysis of bone torsional stiffness and maximum torsion torque of the fracture callus at the middle and late phases of fracture repair further indicate that bone remodeling during the fracture healing process was impaired. Immature woven bone is structurally and mechanically weaker compared with that in mature lamella bone, and the maximum torsion torque value and stiffness strength value are associated with the quality and quantity of new bone.

Our previous cellular and biochemical study provided evidence for the importance of $P D K 1$ in regulating osteoclast formation and bone resorption function in vitro and in vivo (17). The present study further demonstrated, using the tibial bone fracture model in PDK1 cKO mice, that the loss of PDK1, specifically in osteoclasts, impedes fracture healing and repair by delaying cartilaginous callus resorption and remodeling into hard callus in the middle phase and subsequent remodeling of immature woven bone into lamellar bone in the late phase. The impairment in cartilage resorption and bone remodeling was solely due to the decrease of osteoclast formation and activity, as osteoblast bone formation was not affected. These results not only enhance our understanding of the physiological role of $P D K 1$ in osteoclasts, but also provides important clinical implications for the use of anti-resorptive agents, such as bisphosphonates for the treatment of osteolytic conditions. Such anti-resorptive therapies may detrimentally delay fracture healing and repair, and these hypotheses will require validation in future studies. 


\section{Acknowledgements}

Not applicable.

\section{Funding}

This study was supported by the National Natural Science Foundation of China (grant no. 81860402), Guangxi Natural Science Foundation of China (grant no.2017GXNSFAA198073), Guangxi Key Research and Development Project (grant no. GuikeAB17195001), High Level Innovation Team and Excellence Scholars Program of Guangxi High Education Institutions and Guangxi Medical High-level Key Talents Training '139' Program Training Project.

\section{Availability of data and materials}

The datasets used and/or analyzed during the present study are available from the corresponding author on reasonable request.

\section{Authors' contributions}

DX, QuZ and YB performed the experiments, and wrote the manuscript; BC and QiZ analyzed the data; GZ and SZ conceived and designed the study, and reviewed and edited the manuscript. All authors read and approved the final manuscript.

\section{Ethics approval and consent to participate}

The animal experiments were approved by the Animals Ethics Committee of Guangxi Medical University and the Guide for the Care and Use of Laboratory Animals (approval no. 201805021).

\section{Patient consent for publication}

Not applicable.

\section{Competing interests}

The authors declare that they have no competing interests.

\section{References}

1. Kanis JA, Odén A, McCloskey EV, Johansson H, Wahl DA, Cooper C; IOF Working Group on Epidemiology and Quality of Life: A systematic review of hip fracture incidence and probability of fracture worldwide. Osteoporos Int 23: 2239-2256, 2012.

2. Tzioupis $\mathrm{C}$ and Giannoudis PV: Prevalence of long-bone non-unions. Injury 38 (Suppl 2): S3-S9, 2007.

3. Hak DJ, Fitzpatrick D, Bishop JA, Marsh JL, Tilp S, Schnettler R, Simpson $\mathrm{H}$ and Alt V: Delayed union and nonunions: Epidemiology, clinical issues, and financial aspects. Injury 45 (Suppl 2): S3-S7, 2014

4. Einhorn TA: The cell and molecular biology of fracture healing. Clin Orthop Relat Res 355S (Suppl): S7-S21, 1998.

5. Claes L, Recknagel S and Ignatius A: Fracture healing under healthy and inflammatory conditions. Nat Rev Rheumatol 8: 133-143, 2012.

6. Schindeler A, McDonald MM, Bokko P and Little DG: Bone remodeling during fracture repair: The cellular picture. Semin Cell Dev Biol 19: 459-466, 2008.

7. Hadjiargyrou M, Lombardo F, Zhao S, Ahrens W, Joo J, Ahn H, Jurman M, White DW and Rubin CT: Transcriptional profiling of bone regeneration. Insight into the molecular complexity of wound repair. J Biol Chem 277: 30177-30182, 2002.
8. Gerstenfeld LC, Cullinane DM, Barnes GL, Graves DT and Einhorn TA: Fracture healing as a post-natal developmental process: Molecular, spatial, and temporal aspects of its regulation. J Cell Biochem 88: 873-884, 2003.

9. Marsell R and Einhorn TA: The biology of fracture healing. Injury 42: 551-555, 2011.

10. Einhorn TA and Gerstenfeld LC: Fracture healing: Mechanisms and interventions. Nat Rev Rheumatol 11: 45-54, 2015.

11. Ferguson C, Alpern E, Miclau T and Helms JA: Does adult fracture repair recapitulate embryonic skeletal formation? Mech Dev 87: 57-66, 1999.

12. Xin C, Zhi-Ming H, Yasuaki S, Tomoo T and Akira Y: Quantitative study of osteoclastic related factors in the process of bone reconstruction. Hua Xi Kou Qiang Yi Xue Za Zhi 24: 164-165, 2006 (In Chinese).

13. Lin HN and O'Connor JP: Osteoclast depletion with clodronate liposomes delays fracture healing in mice. J Orthop Res 35: 1699-1706, 2017.

14. Cao Y, Mori S, Mashiba T, Westmore MS, Ma L, Sato M, Akiyama T, Shi L, Komatsubara S, Miyamoto K, et al: Raloxifene, estrogen, and alendronate affect the processes of fracture repair differently in ovariectomized rats. J Bone Miner Res 17: 2237-2246, 2002.

15. Flick LM, Weaver JM, Ulrich-Vinther M, Abuzzahab F, Zhang X, Dougall WC, Anderson D, O'Keefe RJ and Schwarz EM: Effects of receptor activator of NFkappaB (RANK) signaling blockade on fracture healing. J Orthop Res 21: 676-684, 2003.

16. Gerstenfeld LC, Sacks DJ, Pelis M, Mason ZD, Graves DT, Barrero M, Ominsky MS, Kostenuik PJ, Morgan EF and Einhorn TA: Comparison of effects of the bisphosphonate alendronate versus the RANKL inhibitor denosumab on murine fracture healing. J Bone Miner Res 24: 196-208, 2009.

17. Xiao D, Zhou Q, Gao Y, Cao B, Zhang Q, Zeng G and Zong S: PDK1 is important lipid kinase for RANKL-induced osteoclast formation and function via the regulation of the Akt-GSK3 $\beta$-NFATc1 signaling cascade. J Cell Biochem: Feb 12, 2020 (Epub ahead of print).

18. Morshed S: Current options for determining fracture union. Adv Med 2014: doi:10.1155/2014/708574

19. Mi M, Jin H, Wang B, Yukata K, Sheu TJ, Ke QH, Tong P, Im HJ, Xiao G and Chen D: Chondrocyte BMP2 signaling plays an essential role in bone fracture healing. Gene 512: 211-218, 2013.

20. van 't Hof RJ, Rose L, Bassonga E and Daroszewska A: Open source software for semi-automated histomorphometry of bone resorption and formation parameters. Bone 99: 69-79, 2017.

21. Abstracts of the Academy of Pediatric Physical Therapy Poster Presentations at the Combined Sections Meeting. Pediatr Phys Ther 31: e31-e69, 2019.

22. Livak KJ and Schmittgen TD: Analysis of relative gene expression data using real-time quantitative PCR and the 2(-Delta Delta C(T)) method. Methods 25: 402-408, 2001.

23. Inada M, Matsumoto C and Miyaura C: Animal models for bone and joint disease. Ovariectomized and orchidectomized animals. Clin Calcium 21: 164-170, 2011 (In Japanese).

24. Frost HM and Jee WS: On the rat model of human osteopenias and osteoporoses. Bone Miner 18: 227-236, 1992.

25. Ghiasi MS, Chen J, Vaziri A, Rodriguez EK and Nazarian A: Bone fracture healing in mechanobiological modeling: A review of principles and methods. Bone Rep 6: 87-100, 2017.

26. Loi F, Córdova LA, Pajarinen J, Lin TH, Yao Z and Goodman SB: Inflammation, fracture and bone repair. Bone 86: 119-130, 2016.

27. Kamimura M, Mori Y, Sugahara-Tobinai A, Takai T and Itoi E: Impaired fracture healing caused by deficiency of the immunoreceptor adaptor protein DAP12. PLoS One 10: e0128210, 2015.

28. Shefelbine SJ, Augat P, Claes L and Beck A: Intact fibula improves fracture healing in a rat tibia osteotomy model. J Orthop Res 23: 489-493, 2005.

29. He LH, Liu M, He Y, Xiao E, Zhao L, Zhang T, Yang HQ and Zhang Y: TRPV1 deletion impaired fracture healing and inhibited osteoclast and osteoblast differentiation. Sci Rep 7: 42385, 2017.

30. McDonald MM, Dulai S, Godfrey C, Amanat N, Sztynda T and Little DG: Bolus or weekly zoledronic acid administration does not delay endochondral fracture repair but weekly dosing enhances delays in hard callus remodeling. Bone 43: 653-662, 2008. 
31. Ota N, Takaishi H, Kosaki N, Takito J, Yoda M, Tohmonda T, Kimura T, Okada Y, Yasuda H, Kawaguchi H, et al: Accelerated cartilage resorption by chondroclasts during bone fracture healing in osteoprotegerin-deficient mice. Endocrinology 150: 4823-4834, 2009.

32. Takahara M, Naruse T, Takagi M, Orui $\mathrm{H}$ and Ogino T: Matrix metalloproteinase-9 expression, tartrate-resistant acid phosphatase activity, and DNA fragmentation in vascular and cellular invasion into cartilage preceding primary endochondral ossification in long bones. J Orthop Res 22: 1050-1057, 2004.

33. Colnot C, Thompson Z, Miclau T, Werb Z and Helms JA: Altered fracture repair in the absence of MMP9. Development 130: 4123-4133, 2003.
34. Li J, Mori S, Kaji Y, Kawanishi J, Akiyama T and Norimatsu H: Concentration of bisphosphonate (incadronate) in callus area and its effects on fracture healing in rats. J Bone Miner Res 15: 2042-2051, 2000.

(i) () () This work is licensed under a Creative Commons Attribution-NonCommercial-NoDerivatives 4.0 International (CC BY-NC-ND 4.0) License. 